\title{
EVALUACIÓN DEL IMPACTO AMBIENTAL DE LA CONSTRUCCIÓN DE UNA PLANTA DE TRATAMIENTO DE AGUAS RESIDUALES. CASO RÍO PINDO CHICO, PUYO, PASTAZA, ECUADOR
}

\section{Evaluation of the environmental impact of the wastewater treatment plant construction. Case Pindo Chico River, Puyo, Pastaza, Ecuador}

\section{Fernando Steven Montero-Vega}

Ingeniero Ambiental. Carrera Ingeniería Ambiental, Facultad Ciencias de la Vida, Universidad Estatal Amazónica, Puyo, Pastaza, Ecuador ORCID: 0000-0002-9099-8737 Correo-e: monterosteven_27@hotmail.com

\section{Carla Stephany Molina-Cedeño}

Ingeniera Ambiental. Carrera Ingeniería Ambiental, Facultad Ciencias de la Vida, Universidad Estatal Amazónica, Puyo, Pastaza, Ecuador ORCID: 0000-0002-1538-3898

Correo-e: amb2015316@uea.edu.ec

\section{Briyidt Maryeli Pillco-Herrera}

Ingeniera Ambiental. Carrera Ingeniería Ambiental, Facultad Ciencias de la Vida, Universidad Estatal Amazónica, Puyo, Pastaza, Ecuador ORCID: 0000-0002-6055-9848

Correo-e: maryelipillco1996@gmail.com

\section{Liliana Bárbara Sarduy-Pereira}

Licenciada en Educación, Especialidad Historia-Filosofía. Docente en Unidad Educativa Fiscomisional Cristóbal Colón, Parroquia Shell, Cantón Mera, Pastaza, Ecuador. ORCID: 0000-0002-7970-3838 Correo-e: lilianasarduy79@gmail.com

\section{Karel Diéguez-Santana}

Candidato a Doctor en Química Industrial, Máster en Ingeniería en Saneamiento Ambiental, docente-investigador de la Carrera Ingeniería Ambiental, Facultad Ciencias de la Vida, Universidad Estatal Amazónica, Puyo, Pastaza, Ecuador ORCID: 0000-0003-4064-0566 Correos-e: karel.dieguez.santana@gmail.com, kdieguez@uea.edu.ec

Recibido: 9/4/2020 • Aprobado: 20/6/2020

Cómo citar: Montero-Vega, F. S., Molina-Cedeño, C. S., Pillco-Herrera, B. M., Sarduy-Pereira, L. B., \& Diéguez-Santana, K. (2020). Evaluación del impacto ambiental de la construcción de una planta de tratamiento de aguas residuales. Caso río Pindo Chico, Puyo, Pastaza, Ecuador. Ciencia, Ambiente y Clima, 3(1), 23-39. Doi: https://doi.org/10.22206/cac.2020.v3i1.pp23-39

\section{Resumen}

El crecimiento de la población, la industrialización, las prácticas agrícolas y la urbanización aumentan la demanda de agua y, por lo tanto, la cantidad de aguas residuales generadas. El propósito de este documento es determinar los principales impactos ambientales de la construcción de la planta de tratamiento de aguas residuales del subsistema Pindo Chico en la ciudad del Puyo, Ecuador. Se recolectó información de línea base, mediante la implementación de listas de chequeo, y para la identificación y evaluación de impactos se confeccionó un diagrama de redes y se

\begin{abstract}
The growth of the population, industrialization, agricultural practices and urbanization increases the demand for water and, therefore, the amount of waste water generated. The purpose of this paper is to determine the main environmental impacts of the wastewater treatment plant construction for the Pindo Chico subsystem in the Puyo city. Baseline information was collected by checklists, and for the identification and evaluation of impacts, a network diagram was made and double-entry matrix structures based on the original Leopold system were used. Seven
\end{abstract}


emplearon estructuras matriciales de doble entrada basadas en el sistema de Leopold original. Se analizaron siete actividades del proyecto y 12 componentes ambientales, y se determinaron 24 interacciones, de las cuales 11 fueron categorizadas como impactos ambientales significativos y dos muy significativos. El componente más afectado es la alteración del suelo, debido a la remoción de la cobertura vegetal, apertura de zanjas, creación de vías de acceso y construcción de la planta. Finalmente, se propusieron medidas correctivas en el plan de manejo ambiental para mitigar, prevenir y monitorear los impactos negativos que se generan.

Palabras clave: evaluación del impacto ambiental; calidad del agua; alcantarillado; saneamiento; agua residual.

\section{Introducción}

El crecimiento de la población, la industrialización, las prácticas agrícolas y la urbanización aumentan la demanda de agua y, por lo tanto, la cantidad de aguas residuales generadas. Varios problemas de contaminación y hacia la salud humana son provocados por la descarga directa de aguas residuales sin ningún proceso de tratamiento (Foley, de Haas, Hartley, \& Lant, 2010).

América Latina, con aproximadamente un tercio de los recursos hídricos del mundo y una media de agua per cápita por año de aproximadamente $22000 \mathrm{~m}^{3}$, es una región con alta disponibilidad de recursos de agua dulce según el Banco Interamericano de Desarrollo (BID, 2018). Sin embargo, solo se trata el $20 \%$ de las aguas residuales municipales (Hernández-Padilla, Margni, Noyola, Guereca-Hernandez, $\&$ Bulle, 2017). Esto hace que la construcción de nuevas instalaciones sean estrategias oportunas para la gestión sostenible de las aguas residuales de la región.

La Plantas de Tratamiento de Aguas Residuales (PTAR) están diseñadas para eliminar los contami- project activities and 12 environmental components were analyzed, a total of the 24 interactions were determined, of which 11 were categorized as significant and two very significant environmental impacts. The most affected component is the soil alteration, due to the vegetation cover removal, the ditches opening, the access routes creation, and the plant construction. Finally, corrective measures in an environmental management plan have been proposed to mitigate, prevent and monitoring the negative impacts they are generated.

Keywords: Environmental impact assessment; water quality; sewers; sanitation; waste water.

nantes de las aguas residuales, producir efluentes que puedan descargarse a cuerpo receptor con un impacto ambiental reducido, cumpliendo con los estándares de descarga locales o nacionales. A pesar de su contribución beneficiosa para el medio ambiente, también generan impactos ambientales durante su construcción, operación y abandono (Morera, Corominas, Rigola, Poch, \& Comas, 2017).

La producción de aguas residuales en Ecuador es un problema al que se ha intervenido de manera ineficaz ya que no se dispone de infraestructuras físicas suficientes. El $90 \%$ de las aguas residuales en Ecuador se descargan en las fuentes de aguas dulces, sin algún tipo de tratamiento, según la Secretaría Nacional del Agua (SENAGUA, 2016). A nivel municipal, se pueden generar derroches de agua por el consumo poblacional y los sistemas sanitarios ineficientes o en mal estado y de esta manera sin tomar en cuenta el volumen ni las condiciones físicas, químicas o biológicas se generan descargas que al final de su ciclo terminan reincorporándose a los cauces naturales sin recibir previo tratamiento (Lahera Ramón, 2010).

La provincia de Pastaza, según los datos del censo poblacional del año 2010, está conformada por 
una población de 83.933 habitantes con un índice de crecimiento poblacional del $3.41 \%$, es la provincia de mayor extensión del país, pero una de las que presenta mayores problemas en los servicios de saneamiento, según el Instituto Nacional de Estadísticas y Censos (INEC, 2010). Las políticas públicas que rigen las competencias de los Gobiernos Autónomos Descentralizados a nivel nacional, establecen la gestión sobre el sistema de red de alcantarillado pluvial y sanitario así como también el tratamiento y disposición final de las aguas municipales; por ende, realizar la ejecución de proyectos como la construcción del subsistema de alcantarillado sanitario y pluvial del río Pindo Chico de la ciudad de Puyo, pueden ser obras importantes para solventar algunos de los problemas ambientales de la zona.

El área de construcción de la planta de tratamiento de aguas residuales (PTAR) presenta ciertas características: desde el inicio de la construcción hasta su abandono se producirá una variedad de impactos que afectarán a los componentes del medio físico, como es el suelo, agua y aire; también se verán afectados los componentes bióticos debido al desbroce y retiro de vegetación. Otro de los impactos más sobresalientes es el componente socioeconómico de las actividades comerciales y/o bienestar público de los sectores intervenidos en el proyecto.

Por ello, de acuerdo con lo descrito anteriormente, este trabajo busca elaborar un estudio de impacto ambiental de dicha construcción, con la finalidad de identificar el grado de perturbación que puede generar hacia el medio físico y ecológico circun- dante al área de influencia de la obra, y de esta manera establecer medidas ambientales que permitan prevenir, controlar, minimizar y mitigar los posibles impactos generados dentro del proyecto.

\section{Materiales y métodos}

\section{Localización}

El proyecto construcción de la Planta de Tratamiento de Aguas Residuales Subsistema Pindo Chico se encuentra ubicado en los terrenos posteriores del Instituto Tecnológico Superior Francisco de Orellana, en el barrio El Dorado de la ciudad del Puyo, entre las coordenadas $\mathrm{X}=833084.30 \mathrm{~m} \mathrm{E} \mathrm{y}$ $\mathrm{Y}=9834507.31 \mathrm{~m} \mathrm{~S}$, cantón Pastaza, provincia de Pastaza. La ciudad del Puyo, según censo del INEC en el año 2010, cuenta con una población de 36.659 hab. Además de poseer un clima agradable que oscila entre 17 y $22^{\circ} \mathrm{C}$ y precipitaciones promedio de $4500 \mathrm{~mm}$ al año, según el Instituto Nacional de Meteorología e Hidrología (INAMHI, 2015). La figura 1 muestra la ubicación geográfica.

El proyecto de alcantarillado pluvial y sanitario, subsistema Pindo Chico, abarcará un área de intervención de 25 hectáreas aproximadamente, mismas que se encuentran distribuidas a lo largo de los barrios: el Dorado, Intipungo, las Palmas, Recreo y Miraflores en los que se realizarán trabajos de tendido de la red de alcantarillado y un área destinada para la construcción de la planta de tratamiento de aguas residuales. 

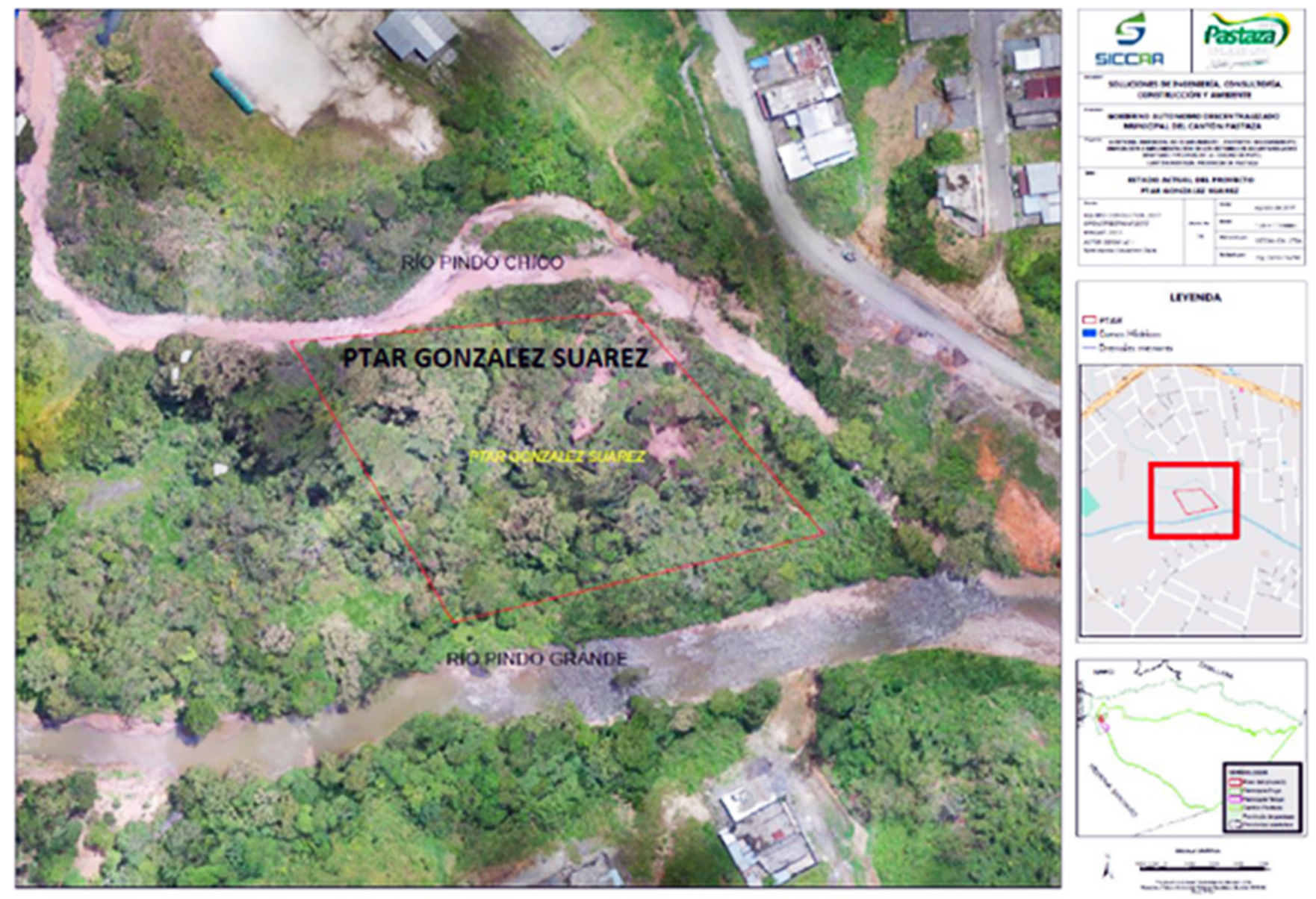

Figura 1. Mapa de ubicación del área de estudio

Fuente: Proyecto Soluciones de Ingeniería, Consultoría, Construcción y Ambiente.

\section{Descripción del proyecto, obra, o actividad}

La ejecución del proyecto de construcción del subsistema de alcantarillado sanitario y pluvial del río Pindo Chico de la ciudad de Puyo, cantón Pastaza, provincia Pastaza comprende una serie de actividades técnicas que abarcan funciones multidisciplinarias, desde la planificación hasta la culminación de la obra, dichos procesos generales de construcción comprenden las etapas presentadas en la figura 2. 


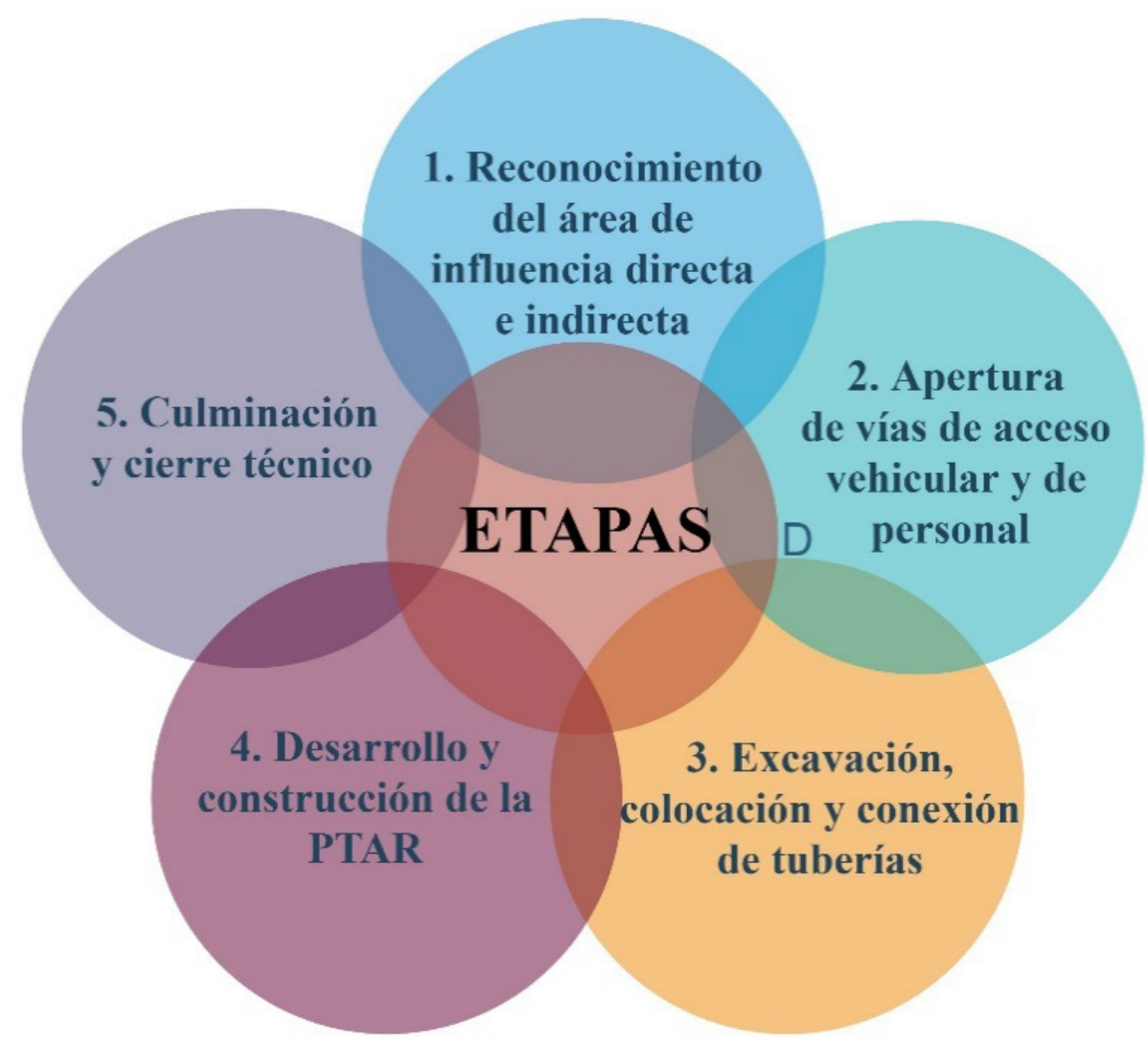

Figura 2. Etapas de la construcción de la PTAR río Pindo Chico Fuente: elaboración propia.

Las etapas son las siguientes:

1. Reconocimiento del área de influencia directa e indirecta del proyecto: el inicio de esta actividad se realizó con la intervención de personal para la limpieza y desbroce de cobertura vegetal presente en el área de influencia de la construcción de la PTAR, además de la identificación de especies florísticas y faunísticas como levantamiento de información primaria de línea base.

2. Apertura de vías de acceso vehicular y de personal para el área de construcción de la PTAR: se realiza el cambio de uso de suelo mediante la introducción de material pétreo y operación de maquinaria pesada para la construcción de la vía de acceso preliminar a la construcción de la planta de tratamiento.
3. Excavación, colocación y conexión de tuberías diferenciadas para el alcantarillado pluvial y sanitario: se intervienen las vías presentes en el plan de conexión de alcantarillado mediante excavación con maquinaria y equipo caminero para la instalación técnica de tubería, así como también el cierre y reparación de vías afectadas.

4. Desarrollo y construcción de la PTAR: se establecen las áreas destinadas a la construcción de las infraestructuras técnicas de acopio de material para la construcción, aparcamiento y mantenimiento de maquinaria y equipos. Inicio de la construcción de la estructura física de la planta de tratamiento de aguas residuales.

5. Culminación y cierre técnico de la obra: se realizan actividades de retiro de escombros y materiales sobrantes de la construcción. 


\section{Criterios analizados en la línea base}

Para el análisis se siguieron los criterios empleados por Soto-Cabrera, Panimboza-Ojeda, Ilibay-Granda, Valverde-Lara y Diéguez-Santana (2020), donde a modo de diagnóstico ambiental se debe alcanzar una comprensión de los ecosistemas y su funcionamiento, así como el grado de afectación que puedan provocar las actividades que se ejecutarán. Estos criterios se basan en los términos de referencias definidos por la autoridad ambiental para cada tipo de actividad específica. En cuanto a la obtención de la línea base, se procedió a recopilar información de estudios ya realizados y se efectuaron visitas de campo a fin de que la información fuese veraz.

Según el Reglamento del Código Orgánico del Ambiente, vigente desde el 2019, la caracterización debe abarcar la descripción del medio físico, biótico y aspectos socioeconómicos y culturales de la población que habita en el área, donde se va a desarrollar el proyecto obra o actividad (Oficial, 2019), por lo que en este estudio se analizaron los tres componentes, tanto para su descripción como para la predicción de los impactos ambientales, asociados con la construcción del sistema de alcantarillado pluvial y sanitario.

\section{Instrumentos de valoración empleados}

Diagrama de redes: para el análisis de los impactos que son generados por la construcción de la Planta de tratamiento de aguas residuales Subsistema Pindo Chico, uno de los instrumentos utilizado fue el diagrama de redes, mismo que nos permitió identificar cómo pueden darse las interacciones entre las acciones de ámbito positivo y las de ámbito negativo de una misma obra, proyecto o actividad, así como lo mencionan (Martínez, Toro, \& León, 2019). Aunque son métodos limitados por la ausencia de ponderaciones y calificaciones de los impactos (Soto-Cabrera, Panimboza-Ojeda, Ilibay-Granda, Valverde-Lara, \& Diéguez-Santana, 2020), pero se consideran adecuados para expresar los impactos ecológicos (Anjaneyulu \& Manickam, 2007). No obstante, para la identificación de factores impac- tantes en este trabajo se emplearon sistemas matriciales, que se expondrán posteriormente.

Matrices causa-efecto: el segundo instrumento utilizado para analizar los impactos generados dentro de la etapa de construcción de la planta de tratamiento fue la matriz de Leopold siendo esta la primera realizada para EIA, misma que relaciona componentes ambientales con una columna de acciones de un proyecto, obra o actividad que generen impactos y de esta manera sintetizar y visualizar dichas interacciones (Pardo Buendía, 2002). Si bien es cierto esta matriz está conformada por entradas en filas y columnas, en este formato pueden distinguir un número total de 8.800 interacciones, las cuales se consideran solamente las que tengan mayor relevancia dentro de la obra y al criterio del evaluador. El desarrollo del análisis de impactos con la matriz de Leopold se realiza mediante una hoja de cálculo del programa Excel, ingresando los datos de los factores ambientales en las filas y las actividades dentro de las columnas, permitiéndonos identificar las principales interacciones que surgen a criterio del evaluador para, posteriormente, pasar a la fase de ponderación.

Dentro de los parámetros a evaluar de la matriz de Leopold se analiza la magnitud e importancia (Mora-Barrantes, Molina-León, \& Sibaja-Brenes, 2016), que establecen un grado de significancia de los impactos identificados en una nueva jerarquización. Para la determinación de la importancia de las interacciones identificadas en la matriz se aplicó la ecuación 1.

$$
\begin{gathered}
\operatorname{Imp}=\mathrm{W}_{\mathrm{e}}^{*} \text { Extensión }+\mathrm{W}_{\mathrm{d}}^{*} \text { Duración }+ \\
\mathrm{W}_{\mathrm{r}}^{*} \text { Reversibilidad }
\end{gathered}
$$

Donde:

$$
\begin{aligned}
& \text { Imp }=\text { Importancia } \\
& \text { We }=\text { Peso Extensión }(0.30) \\
& \text { Wd }=\text { Peso de Duración }(0,30) \\
& \text { Wr }=\text { Peso de Reversibilidad }(0.35)
\end{aligned}
$$


Los valores de extensión, duración y reversibilidad del proyecto son tomados de la tabla 1 , de acuerdo con la subjetividad del evaluador. Asimismo, para determinar la magnitud del impacto se realiza con los parámetros ubicados en la tabla 2, donde se consideran como puntos importantes el nivel de intensidad del proyecto y la afectación que va a generar de manera directa e indirecta en el área empleada.

Tabla 1. Criterios de puntuación de la importancia y valores asignados

\begin{tabular}{|c|c|c|c|c|c|}
\hline Características & \multicolumn{5}{|c|}{ Puntuación } \\
\cline { 2 - 6 } & 1.0 & 2.5 & 5.0 & 7.5 & 10.0 \\
\hline Extensión & Puntual & Particular & Local & Generalizada & Regional \\
\hline Duración & Esporádica & Temporal & Periódica & Recurrente & Permanente \\
\hline Reversibilidad & $\begin{array}{c}\text { Completamente } \\
\text { Reversible }\end{array}$ & $\begin{array}{c}\text { Medianamente } \\
\text { Reversible }\end{array}$ & $\begin{array}{c}\text { Parcialmente } \\
\text { Reversible }\end{array}$ & $\begin{array}{c}\text { Medianamente } \\
\text { Reversible }\end{array}$ & $\begin{array}{c}\text { Completamente } \\
\text { Irreversible }\end{array}$ \\
\hline
\end{tabular}

Fuente: elaboración propia.

Tabla 2. Criterios de puntuación de la magnitud y valores asignados

\begin{tabular}{|c|c|c|}
\hline \multicolumn{2}{|c|}{ Magnitud } \\
\hline Calificación & Intensidad & Afección \\
\hline 1 & Baja & Baja \\
\hline 2 & Baja & Media \\
\hline 3 & Baja & Alta \\
\hline 4 & Media & Baja \\
\hline 5 & Media & Media \\
\hline 6 & Media & Alta \\
\hline 7 & Alta & Baja \\
\hline 8 & Alta & Alta \\
\hline 9 & Alta & Alta \\
\hline 10 & Muy alta & \\
\hline
\end{tabular}

Fuente: elaboración propia. 
De esta manera, una vez obtenido el valor de magnitud se procede a calcular el Valor de Impacto (VI), mediante la ecuación 2.

Valor de impacto $(\mathrm{VI})= \pm$

(Importancia x Magnitud) $^{0.5}$

[Ec. 2]

A partir del valor del impacto, se categorizaron en: Altamente significativo $(>-7)$, Significativo $(-4,5$ a -7$)$, Despreciable $(<-4,5)$ y Beneficioso $(>0)$.

\section{Descripción de los aspectos conceptuales del Plan de Manejo de Ambiental}

Se conoce a los planes de manejo ambiental con siglas (PMA) como una herramienta dirigida para el control, mitigación, corrección o compensación de los impactos ambientales que son generados en cada fase de ejecución de toda clase de obra, proyecto o actividad (Lara \& Castro Valencia, 2016). La correcta identificación de riesgos se conforma como una pieza clave para la predicción de accidentes y determinar la gestión óptima dentro de todos los niveles de un proyecto, y de esta manera aumentar la veracidad en la toma de decisiones (Cruz Virosa, Filgueiras Sainz de Rozas, Sorinas Gonzáles, Cabello Eras, \& Fernández Pérez, 2016), con la finalidad de accionar técnica y estratégicamente contra los impactos ambientales significativos y disminuir sus efectos en el ambiente.

De acuerdo con lo estipulado por el Ministerio del Ambiente en el Código Orgánico Ambiental dentro del territorio ecuatoriano, el administrador de una actividad, obra o proyecto, que genere perturbaciones en el ambiente, debe realizar la identificación de los impactos que se promuevan en función de las fases y actividades que realice, para que $a$ posteriori detalle las correspondientes medidas correctivas. Recientemente, en el artículo 435, el Reglamento del Código Orgánico del Ambiente (Oficial, 2019) define que: "El plan de manejo ambiental es el documento que contiene las acciones o medidas que se requieren ejecutar para prevenir, evitar, mitigar, controlar, corregir, compensar, restaurar y reparar los posibles impactos ambientales negativos según corresponda, al proyecto, obra o actividad". El mismo incluye que "el plan de manejo ambiental según la naturaleza del proyecto, obra o actividad contendrá los siguientes sub-planes, considerando los aspectos ambientales, impactos y riesgos identificados".

En el análisis de los impactos generados por la construcción de la PTAR subsistema Pindo Chico y en cumplimiento de lo estipulado por la Autoridad Ambiental Nacional se consideraron acciones para cuatro de los sub-planes, en este caso: Plan de prevención y mitigación de impactos, Plan de manejo de desechos, Plan de Rehabilitación y Plan de cierre y abandono.

\section{Resultados y discusión}

\section{Diagnóstico de línea base}

En la tabla 3 se puede observar el estado actual de los principales componentes ambientales antes de la ejecución de la obra, obtenidos de la línea base. Entre los principales aspectos de la línea base, el componente suelo se encuentra en buen estado, con gran cantidad de capa fértil, este componente se verá afectado directamente con la acumulación de desperdicios, remoción y compactación por la actividad de construcción. 


\section{Evaluación del impacto ambiental de la construcción de una planta de tratamiento de aguas residuales. Caso río Pindo Chico, Puyo, Pastaza, Ecuador}

Tabla 3. Estado actual de los principales componentes de la línea base

\begin{tabular}{|c|c|}
\hline $\begin{array}{l}\text { Componente } \\
\text { ambiental }\end{array}$ & Estado actual \\
\hline Calidad del aire & Buena (no hay actividad industrial/baja fluidez de tránsito vehicular) \\
\hline Ruido & No supera los $50 \mathrm{~dB}$ \\
\hline Calidad del suelo & $\begin{array}{l}\text { Capa fértil. Uso de suelo: zona residencial, con presencia de cobertura vegetal } \\
\text { secundaria. Textura moderadamente gruesa de orden Inceptisol, suborden Andept, } \\
\text { Grangrupo Hydrandept. }\end{array}$ \\
\hline Calidad del agua & $\begin{array}{l}\text { Ligeramente contaminada. Nivel de turbidez elevado, presencia de sólidos } \\
\text { suspendidos, alteración en el pH, y con abundante presencia de materia orgánica. }\end{array}$ \\
\hline Paisaje & Bosque secundario, con alteración de edificaciones. \\
\hline Flora & $\begin{array}{l}\text { Nativa/Alterada. Ecosistema de bosque siempre verde piemontano, ligera presencia } \\
\text { de flora nativa, alteración del ecosistema por construcciones. Especies más } \\
\text { destacadas: Pambil (Iriartea deltoidea) familia Arecaceae, Guarumo (Cecropia peltata) } \\
\text { familia Cecropiaceae, Pigüe (Pillolesta discolor) familia Asteraceae, Guaba bejuco } \\
\text { (Inga edulis) familia Mimosaceae. }\end{array}$ \\
\hline Fauna & $\begin{array}{l}\text { Pocas especies silvestres. Especies terrestres destacadas: monos de bolsillo (Cebuella } \\
\text { pygmaea), gallinazos (Coragyps atratus) y buglas (Psarocolius decumanus); ejemplares } \\
\text { de insectos pertenecientes a los lepidópteros, coleópteros, dípteros, entre otros. } \\
\text { Especies acuáticas destacadas: cardúmenes de peces con longitudes no mayor a } 2 \mathrm{~cm} \text {. }\end{array}$ \\
\hline Sistema económico & Actividades de comercio, microempresa y agricultura. \\
\hline Educación & Media, nivel educacional de la población, medio. \\
\hline Salud & $\begin{array}{l}\text { Media. Enfermedades derivadas de la contaminación del agua. Año, 2015: } \\
\text { parasitosis ( } 101 \text { casos), diarrea ( } 71) \text {, enfermedades infecciosas (48) y otras de carácter } \\
\text { respiratorio y causadas por la contaminación por vectores o mala disposición de la } \\
\text { basura y aguas servidas. }\end{array}$ \\
\hline $\begin{array}{l}\text { Sistema de } \\
\text { alcantarillado }\end{array}$ & Ineficiente, no existe. \\
\hline Empleo local & Medio (predomina comercio, microempresa, agricultura y ganadería) \\
\hline
\end{tabular}

Fuente: elaboración propia. 


\section{Diagrama de redes}

En la figura 3 se presenta el diagrama de redes utilizado para identificar las actividades más impactantes. Esta metodología es una herramienta que permite tener una idea más clara de cuáles son las actividades y componentes con los que se puede trabajar en la matriz de evaluación. De esta manera, se puede identificar que de la actividad central "construcción de la PTAR Pindo Chico" se desglosan dos impactos principales: el cambio en la estructura del ecosistema (negativo) y tratamiento de aguas servidas (positivo); al seguir desglosando el diagrama determinamos que muchos de los impactos negativos tienen incidencia en los positivos y viceversa, como es el caso de la salud humana, que por la construcción de campamentos se generan residuos, y si no son manejados de una manera adecuada pueden proliferar enfermedades. Otro criterio que se puede evidenciar en el diagrama está relacionado con el desarrollo de la sociedad y la calidad del agua, pues el aumento demográfico y las actividades productivas pueden perjudicar su calidad; consideradas en Ecuador como las principales causas de la contaminación del agua (Trejo, Chimbo y Erazo, 2015).

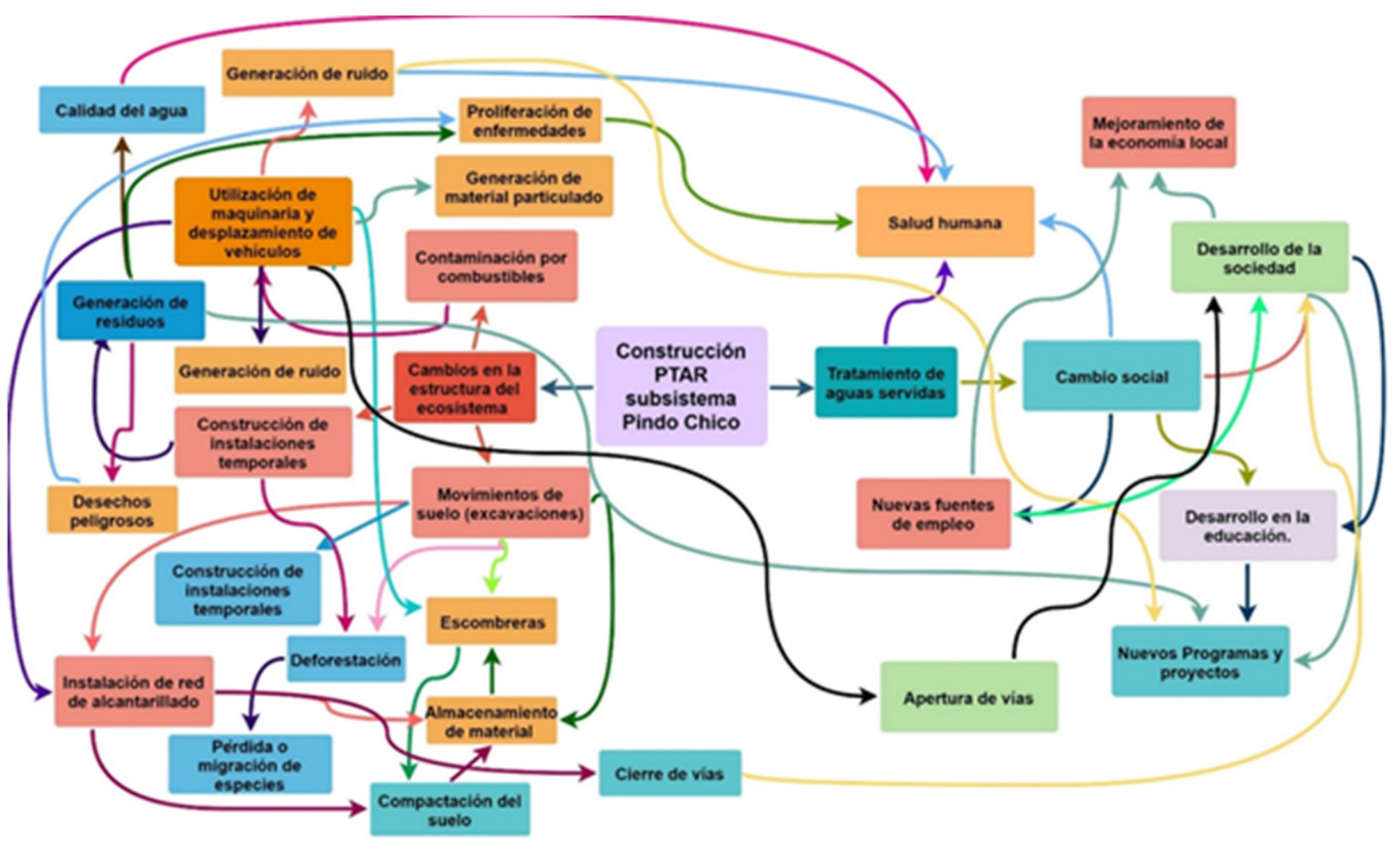

Figura 3. Diagrama de interacción de los impactos ambientales de la construcción de la PTAR Pindo Chico Fuente: elaboración propia. 


\section{Evaluación del impacto ambiental de la construcción de una planta de tratamiento de aguas}

residuales. Caso río Pindo Chico, Puyo, Pastaza, Ecuador

\section{Matriz de Leopold}

La tabla 4 muestra la identificación de las interacciones entre acciones y componentes ambientales.

Tabla 4. Identificación de interacciones entre las actividades del proyecto y los factores impactados

\begin{tabular}{|c|c|c|c|c|c|c|c|c|c|}
\hline & & & $\begin{array}{l}\text { Sociali- } \\
\text { zación } \\
\text { proyecto }\end{array}$ & $\begin{array}{l}\text { Inventario } \\
\text { Flora y } \\
\text { Fauna }\end{array}$ & $\begin{array}{l}\text { Desbroce de } \\
\text { cobertura } \\
\text { vegetal }\end{array}$ & $\begin{array}{l}\text { Apertura } \\
\text { de vías- } \\
\text { Operación de } \\
\text { maquinaria }\end{array}$ & $\begin{array}{l}\text { Excavación } \\
\text { y cambio de } \\
\text { la estructura } \\
\text { del suelo }\end{array}$ & $\begin{array}{l}\text { Ruptura del } \\
\text { pavimento }\end{array}$ & $\begin{array}{l}\text { Repavimen- } \\
\text { tación de } \\
\text { vías afectadas }\end{array}$ \\
\hline \multirow[t]{6}{*}{$\begin{array}{l}\text { Medio } \\
\text { Físico }\end{array}$} & \multirow[t]{3}{*}{ Suelo } & $\begin{array}{l}\text { Calidad del } \\
\text { suelo }\end{array}$ & & & $\mathrm{X}$ & $\mathrm{X}$ & $X$ & & \\
\hline & & $\begin{array}{l}\text { Presencia } \\
\text { de residuos } \\
\text { sólidos } \\
\text { (Escombros) }\end{array}$ & & & & $X$ & $\mathrm{X}$ & $X$ & \\
\hline & & Uso de suelo & & & & $\mathrm{X}$ & & & \\
\hline & Agua & $\begin{array}{l}\text { Calidad del } \\
\text { agua }\end{array}$ & & & & & & & $\mathrm{X}$ \\
\hline & \multirow[t]{2}{*}{ Aire } & $\begin{array}{l}\text { Calidad del } \\
\text { aire }\end{array}$ & & & & $\mathrm{X}$ & $\mathrm{X}$ & & \\
\hline & & $\begin{array}{l}\text { Ruido y } \\
\text { vibraciones }\end{array}$ & & & & $\mathrm{X}$ & $\mathrm{X}$ & $\mathrm{X}$ & $\mathrm{X}$ \\
\hline \multirow[t]{3}{*}{$\begin{array}{l}\text { Medio } \\
\text { Biótico }\end{array}$} & Flora & $\begin{array}{l}\text { Cobertura } \\
\text { vegetal }\end{array}$ & & $\mathrm{X}$ & $\mathrm{X}$ & & $\mathrm{X}$ & & \\
\hline & \multirow[t]{2}{*}{ Fauna } & $\begin{array}{l}\text { Fragmentación } \\
\text { del hábitat }\end{array}$ & & $\mathrm{X}$ & $\mathrm{X}$ & $\mathrm{X}$ & & & \\
\hline & & $\begin{array}{l}\text { Migración de } \\
\text { especies }\end{array}$ & & & $\mathrm{X}$ & & & & \\
\hline \multirow{3}{*}{$\begin{array}{l}\text { Medio } \\
\text { Socioeco- } \\
\text { nómico y } \\
\text { cultural }\end{array}$} & \multirow[t]{2}{*}{$\begin{array}{l}\text { Aspectos } \\
\text { culturales }\end{array}$} & $\begin{array}{l}\text { Salud y } \\
\text { seguridad }\end{array}$ & & & & $\mathrm{X}$ & & & \\
\hline & & Empleo & $\mathrm{X}$ & & & & & & \\
\hline & $\begin{array}{l}\text { Facilidades y } \\
\text { actividades } \\
\text { humanas }\end{array}$ & $\begin{array}{l}\text { Acceso y } \\
\text { movilidad }\end{array}$ & & & & & & $\mathrm{X}$ & $\mathrm{X}$ \\
\hline
\end{tabular}

Fuente: elaboración propia. 
Como se puede apreciar, se obtuvieron 24 interacciones principales, provenientes de las siete actividades consideradas y los 12 factores ambientales evaluados. Estas interacciones se ponderaron y clasificaron según su impacto. En resumen, fueron identificados 2 impactos muy significativos, 13 significativos, 5 despreciables y 4 beneficiosos. Esta categorización de los impactos ha sido sugerida por Pardo Buendía (2002). En la figura 3 se puede observar con detalle cada una de las principales actividades del proyecto, con la cantidad y grado de impacto que generan.

La apertura de nuevas vías y operación de maquinaria es de las actividades más impactantes, aunque la repavimentación y la excavación y cambio de la estructura del suelo son las dos actividades que contribuyen con los impactos más negativos, en este caso, muy significativos. De igual forma, el desbroce de cobertura vegetal también conlleva un peso importante y negativo al medio ambiente, puesto que según Mena y Segarra (2016) esta actividad en proyectos de construcción en la Amazonia, siempre va a tener una contribución negativa, dado la presencia de especies vegetales y animales nativas, por lo que se deben considerar la implementación de protocolos enfocados a minimizar esta afectación.

\section{Actividades del proyecto vs grado de impacto ambiental}

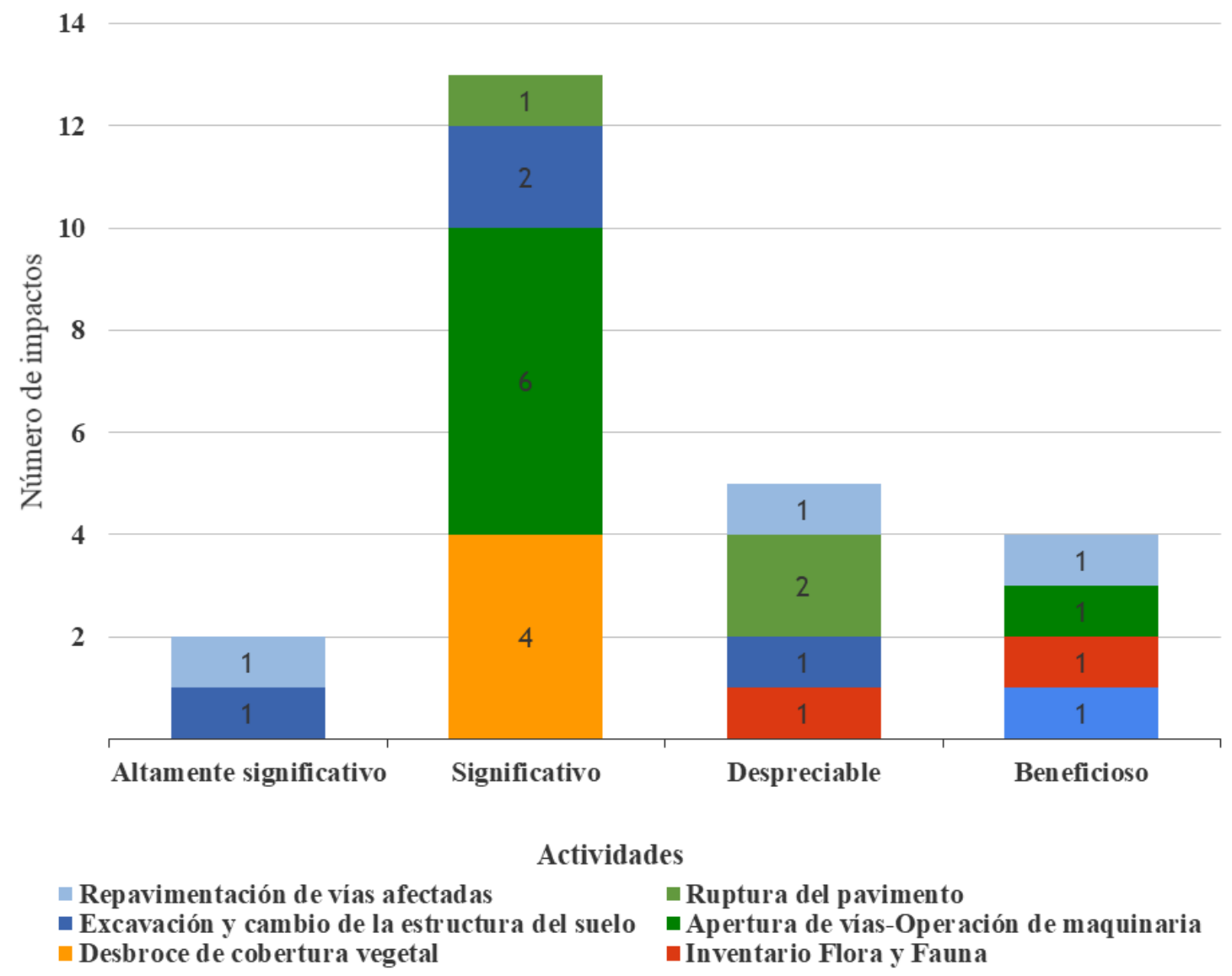

Figura 3. Actividades del proyecto vs. grado de impacto ambiental

Fuente: elaboración propia.

34 | Ciencia, Ambiente y Clima 2020; 3(1, enero-junio): 23-39 
Por su parte, la figura 4 muestra la afectación de los diversos factores ambientales considerados, donde sobresale el componente suelo, que será gravemente afectado como consecuencia de la remoción de la cobertura vegetal, apertura de zanjas, creación de vías de acceso, así como la excavación y cambio de la estructura del suelo con material pétreo y concreto, que es uno de los más severo de esta actividad. Este impacto es frecuente en obras civiles, y en construcción de PTAR (Mena \& Segarra, 2016; Morera, Corominas, Rigola, Poch, \& Comas, 2017; Morera, Remy, Comas, \& Corominas, 2016).

\section{Factores ambientales afectados según el grado y número de impactos \\ Altamente significativo \\ - Despreciable \\ - Significativo \\ - Ben eficioso}

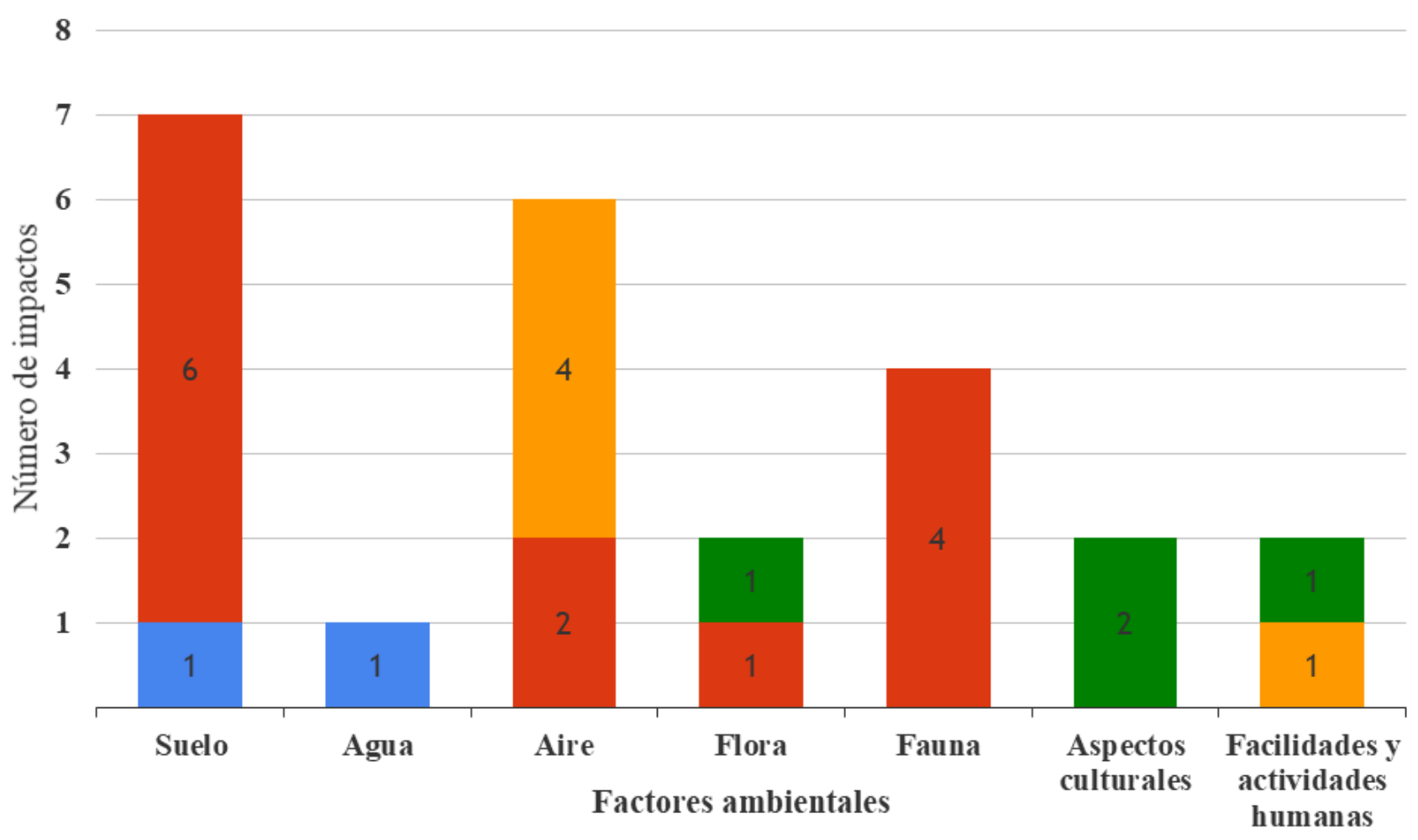

Figura 4. Factores ambientales del proyecto vs. grado de impacto ambiental Fuente: elaboración propia.

Dentro de la categoría de impactos beneficiosos resaltan los aspectos culturales y las facilidades de la actividad humana que se verá beneficiada por el empleo durante la construcción, también por el mejoramiento de la calidad de vida una vez terminada la PTAR, y que la contaminación de los cauces superficiales disminuirá por verter el agua tratada.

\section{Plan de Manejo Ambiental (PMA)}

Con la finalidad de controlar los impactos generados en las actividades dentro de la construcción de la planta de tratamiento de aguas residuales, se determinaron los siguientes subplanes de manejo ambiental distribuidos de la siguiente manera: 
Se estableció un Plan de prevención y Mitigación para las actividades de desbroce, limpieza y cobertura vegetal, mediante medidas preventivas para evitar la tala innecesaria de especies arbóreas de gran dimensión, sensibles y amenazadas dentro del área de estudio. Además, para la actividad de excavación y cambio de la estructura del suelo con material pétreo y concreto, que afectan directamente al suelo y a la calidad del aire. Para ello, se busca implementar medidas de remoción inmediata de residuos de construcción durante su ejecución, para evitar factores externos, pues como plantean Hernández-Columbié y Ulloa-Carcasés (2014), luego de cada actividad se deberán retirar los residuos que se generan de forma explícita de la obra, y como medida de prevención de derrames, se utilizarán cobertores plásticos para cubrir el material, y de esta forma evitar el arrastre de partículas de polvo por el viento.

En el segundo plan generado, de acuerdo con las características de las actividades realizadas durante la construcción de la PTAR, es el plan de manejo de desechos, que tiene la finalidad de aprovechar la mayor parte de material y disponer los desechos y escombros sin utilidad en áreas autorizadas bajo protocolos de control municipal. Esta acción debe basarse en el diseño efectivo de construcción y control de desechos de los mismos, con la finalidad de mejorar la eficiencia (Ajayi et al., 2017). Entre las principales actividades en las que se basa este plan, se tienen: la apertura de vías de acceso e ingreso y operación de maquinaria pesada, materiales de construcción, insumos y equipos; excavación y cambio de estructura del suelo con material pétreo; y la ruptura del pavimento excavación y desalojo del suelo.

El tercer plan desarrollado es el de Plan de Rehabilitación, en el mismo se consideraron las acciones correspondientes para tratar de contrarrestar los impactos generados por el desbroce y la limpieza de la cobertura vegetal, la excavación y cambio de la estructura del suelo con material pétreo, que alteran la calidad del suelo y fragmentan el hábitat del área circundante de estudio. Para estas actividades se determina que, luego de finalizadas las actividades de construcción, se deberán implementar programas de reforestación. En caso de existir la presencia de materiales pétreos, concreto, hormigón, entre otros, se removerán y reemplazarán por un suelo nuevo, cuya finalidad es que las especies de flora identificadas para la rehabilitación puedan desarrollarse de mejor manera, así como también la estructura del suelo se conforme de manera natural (Hartig, Sanders, Wyma, Boase \& Roseman, 2018).

Para dar una buena imagen del proyecto y evitar impactos significativos directa e indirectamente a la calidad del suelo y agua, se busca realizar un cierre técnico de operaciones y el cese de actividades, aplicando el desmantelamiento de equipos y maquinaria, control de acceso y la limpieza total del área intervenida del proyecto, una vez culminada la repavimentación de las vías afectadas.

\section{Consideraciones generales}

Como plantea Fuentes-Bargues (2018), los impactos identificados y evaluados dependen de la tecnología utilizada, la ubicación de la planta, el entorno receptor del agua tratada y si el agua tratada se reutiliza. El suelo es de los componentes más afectados, es un impacto permanente que comienza con la construcción y continúa durante la operación. También ocurren derrames de aceites y restos de combustible que inciden sobre el terreno, incluso, pueden perturbar las aguas superficiales por las lluvias, escorrentías, que pueden arrastrarlos hasta las orillas de los ríos (las PTAR están cercanas a cauces de agua superficiales).

Otra afectación que pueden recibir los cauces superficiales es el arrastre de sedimentos, materia orgánica del suelo, donde cantidades de nitrógeno pueden 


\section{Evaluación del impacto ambiental de la construcción de una planta de tratamiento de aguas residuales. Caso río Pindo Chico, Puyo, Pastaza, Ecuador}

causar la eutrofización de cursos de aguas superficiales (Diéguez-Santana, Arteaga-Pérez, Casas Ledón \& Rodríguez Rico, 2013; Diéguez-Santana, Casas-Ledón, Loureiro Salabarria, Pérez-Martínez \& Arteaga-Pérez, 2020).

Durante la fase de construcción el ruido de la maquinaria puede afectar a los trabajadores, pues en este caso no existen cargas sonoras elevadas en el medio circundante; también, pueden perturbar la calidad del aire, especialmente durante los trabajos de movimiento de tierras como consecuencia de la emisión de polvo y motores de gas de la maquinaria.

Si se analizan los impactos derivados por los materiales empleados y los recursos consumidos en la fase de construcción tienen una elevada incidencia; el material de tubería representa del 30 al $60 \%$ de los impactos ambientales de la construcción y un 33 al $74 \%$ de los impactos de una renovación de un sistema de alcantarillado, en un estudio del análisis de ciclo de vida realizado por Morera, Remy, Comas y Corominas (2016). Las características específicas del sitio pueden influir en la construcción y renovación de los sistemas de alcantarillados, pues cuando la construcción requiere colocación de asfalto en la parte superior de las zanjas abiertas, incrementan los impactos ambientales del 20 al $55 \%$, y la presencia de suelo rocoso, a diferencia del suelo compacto o blando, puede aumentar los impactos del 9 al 34 \% (Morera, Remy, Comas \& Corominas, 2016).

\section{Conclusiones}

La realización de la evaluación de impacto ambiental en todas las fases de un proyecto, obra o actividad dentro del sector de la construcción es de gran importancia, pues garantiza el buen uso del recurso, previene la ejecución de actividades con bajo, mediano y alto impacto para el ambiente, lo que permite realizar estructuras en armonía con los ecosistemas. El tratamiento de las aguas residuales es un proceso necesario para el medio ambiente, por lo que la construcción y operación de estas instalaciones es necesaria, aunque genera varios impactos negativos al ambiente. Los principales impactos identificados del proyecto de construcción de la Planta de tratamiento de aguas residuales subsistema Pindo Chico son: la alteración del suelo, por la remoción de la cobertura vegetal, apertura de zanjas, vías de acceso y construcción de la planta. Adicionalmente, impactos como afectación de la calidad del agua, y el aire tienen consecuencias negativas. En el caso de los impactos positivos, esta obra contribuirá a mejorar la salud de la población y la calidad de las fuentes hídricas que circundan en el área de influencia, directa e indirecta, del proyecto, en la ciudad del Puyo, pues ayuda a las que se descargan las aguas residuales sin ningún tipo de tratamiento previo. Finalmente, las medidas de manejo propuestas pueden contribuir a mitigar, prevenir y dar un seguimiento a los impactos negativos que se generan.

\section{Agradecimientos}

Los autores agradecen a los ejecutantes del proyecto de construcción de la Planta de Tratamiento de Aguas Residuales Subsistema Pindo Chico por el acceso a las instalaciones e información proporcionada, además a la Universidad Estatal Amazónica por propiciar esta investigación.

\section{Referencias}

Ajayi, S. O., Oyedele, L. O., Akinade, O. O., Bilal, M., Alaka, H. A., Owolabi, H. A., \& Kadiri, K. O. (2017). Attributes of design for construction waste minimization: A case study of waste-to-energy project. Renewable Sustainable Energy Reviews, 73, 1333-1341. Doi:10.1016/j.rser.2017.01.084

Anjaneyulu, Y., \& Manickam, V. (2007). Chapter 2. EIA Methodologies In Environmental Impact Assessment Methodologies (2nd Ed.). Hyderabad, India: BS Publications. 
Banco Interamericano de Desarrollo -BID. (2018). Informe Regional América Latina y el Caribe. Resumen ejecutivo 2018. Santiago de Chile. Recuperado de: https://www.cepal.org/sites/ default/files/news/files/informe_regional_ america_latina_y_caribe.pdf

Cruz Virosa, I., Filgueiras Sainz de Rozas, M. L., Sorinas Gonzáles, L., Cabello Eras, J. J., \& Fernández Pérez, L. (2016). Gestión comparada del riesgo en el control de la contaminación atmosférica de Generadores de Vapor. Ingeniería Energética, 37(3), 195-206.

Diéguez-Santana, K., Arteaga-Pérez, L. E., Casas Ledón, Y., \& Rodríguez Rico, I. L. (2013). Análisis de ciclo de vida y caracterización ambiental en una industria alimenticia. Revista Centro Azúcar, 40, 52-58.

Diéguez-Santana, K., Casas-Ledón, Y., Loureiro Salabarria, J. A., Pérez-Martínez, A., \& Arteaga-Pérez, L. E. (2020). A life cycle assessment of bread production: A Cuban case study. Journal of Environmental Accounting and Management, 8(2), 125-137. Doi:10.5890/ JEAM.2020.06.002

Foley, J., de Haas, D., Hartley, K., \& Lant, P. (2010). Comprehensive life cycle inventories of alternative wastewater treatment systems. Water Research, 44(5), 1654-1666. Doi:10.1016/j. watres.2009.11.031

Fuentes-Bargues, J. L. (2018). Review of the environmental impact assessment process of wastewater treatment plants in Spain. Environment Protection Engineering, 44(4), 23-41. Doi:10.5277/epe180402

Hartig, J., Sanders, C., Wyma, R., Boase, J. C., \& Roseman, E. (2018). Habitat rehabilitation in the Detroit River Area of Concern. Aquatic ecosystem health management, 21(4), 458-469. Doi:10.1080/14634988.2018.1536437

Hernández-Columbié, T., \& Ulloa-Carcasés, M. (2014). Impacto ambiental de la ampliación de una presa de colas de la industria cubana del níquel. Minería y geología, 30(3), 33-48.
Hernández-Padilla, F., Margni, M., Noyola, A., Guereca-Hernandez, L., \& Bulle, C. (2017). Assessing wastewater treatment in Latin America and the Caribbean: Enhancing life cycle assessment interpretation by regionalization and impact assessment sensibility. Journal of Cleaner Production, 142, 2140-2153. Doi:10.1016/j. jclepro.2016.11.068

INAMHI. (2015). Anuarios Meteorológicos. No 52. Quito, Ecuador. Recuperado de: http://www. serviciometeorologico.gob.ec/wp-content/ uploads/anuarios/meteorologicos/Am\%20 2012.pdf

Instituto Nacional de Estadísticas y Censo -INEC. (2010). Resultados del Censo de población y vivienda en el Ecuador 2010. Fascículo Provincial Pastaza. Recuperado de https:// www.ecuadorencifras.gob.ec/wp-content/ descargas/Manu-lateral/Resultados-provinciales/pastaza.pdf

Lahera Ramón, V. (2010). Infraestructura sustentable: las plantas de tratamiento de aguas residuales. Quivera. Revista de Estudios Territoriales, 12(2), 58-69.

Lara, Y. A., \& Castro Valencia, D. M. (2016). Los Planes de Manejo Ambiental (PMA): una herramienta de control a los impactos ambientales que generan la instalación de redes servicios públicos domiciliarios en Colombia. Tecnogestión: Una mirada al ambiente, 13(1).

Martínez, L. F., Toro, J., \& León, C. J. (2019). A complex network approach to environmental impact assessment. Impact Assessment Project Appraisal, 37(5), 407-420. Doi:10.1080/146 15517.2018.1552442

Mena, A. A., \& Segarra, E. (2016). Diseńo del proyecto ecoturístico Shalcani y valoración de impactos ambientales durante la fase constructiva, Misahuallí, Napo. Siembra, 3(1), 147-154.

Mora-Barrantes, J. C., Molina-León, Ó. M., \& Sibaja-Brenes, J. P. (2016). Aplicación de un método para evaluar el impacto ambiental de 
proyectos de construcción de edificaciones universitarias. Revista Tecnología en Marcha, 29(3), 132-145.

Morera, S., Corominas, L., Rigola, M., Poch, M., \& Comas, J. (2017). Using a detailed inventory of a large wastewater treatment plant to estimate the relative importance of construction to the overall environmental impacts. Water Research, 122, 614-623. Doi:10.1016/j.watres.2017.05.069

Morera, S., Remy, C., Comas, J., \& Corominas, L. (2016). Life cycle assessment of construction and renovation of sewer systems using a detailed inventory tool. The International Journal of Life Cycle Assessment, 21(8), 1121-1133. Doi:10.1007/s11367-016-1078-9

Oficial, R. (2019). Reglamento del Código Orgánico del Ambiente Año III - No 507. Decreto Ejecutivo No. 752 de 21 de mayo de 2019. Quito, Ecuador. Recuperado de: http://apel. ec/biblioteca/decreto-752-2019-reglamento-al-codigo-organico-del-ambiente/
Pardo Buendía, M. (2002). La evaluación del impacto ambiental y social para el siglo XXI: teorías, procesos, metodología. Madrid, España: Editorial Fundamentos.

SENAGUA. (2016). Estrategia Nacional de Agua Potable y Saneamiento. Ecuador. Recuperado de: https://www.agua.gob.ec/wp-content/ uploads/downloads/2016/07/REVISTA-SENAGUA.compressed.pdf

Soto-Cabrera, A. I., Panimboza-Ojeda, A. P., Ilibay-Granda, C. G., Valverde-Lara, C. R., \& Diéguez-Santana, K. (2020). Impacto ambiental de la operación del Centro de faenamiento de la ciudad de Puyo, Pastaza, Ecuador. Prospectiva, 18(1), 60-68. Doi:10. 15665/rp.v18i1.2101

Trejo, C. A., Chimbo, K. S. O., \& Erazo, R. A. M. (2015). El crecimiento poblacional y su impacto la contaminación ambiental. Revista Contribuciones a las Ciencias Sociales, (27). 